\title{
COMPUTING LOWER BOUNDS OF $\mu$-VALUES FOR A CLASS OF ROTARY ELECTRICAL MACHINES
}

\author{
MUTTI-UR REHMAN*, M. FAZEEL ANWAR \\ Department of Mathematics, Sukkur IBA University, 65200 Sukkur-Pakistan \\ *Corresponding author: mutti.rehman@iba-suk.edu.pk
}

\begin{abstract}
In this article we present the computations of lower bounds of well-known mathematical quantity in control theory known as structured singular value for a family of structured matrices obtained for a DC Motor, that is an electrical machine. The comparison of lower bounds with the well-known MATLAB function mussv is studied. The structured singular values provide an important tool to synthesize robustness as well as analyze performance and stability of feedback systems.
\end{abstract}

\section{INTRODUCTION}

The Structured Singular Value (SSV) [1] is an important and versatile tool in system theory which allows one to address a central problem in control systems i.e. to analyze the stability of a system. The class of structures addressed by the SSV is very generic and covers almost all kinds of parametric perturbations that can be incorporated into the control system via real or complex linear fractional transformations (LFTs). We refer the interested readers to see [2 - 9] and the references there in for more examples and applications of SSV.

The exact computations of SSV are NP hard [10]. There are however several algorithms available in literature to compute lower and upper bounds for SSV. An upper bound of SSV guaranties the robust stability, while the lower bounds of SSV provides information about instability of linear closed loop systems.

Received 2018-08-04; accepted 2018-10-06; published 2019-01-04.

2010 Mathematics Subject Classification. 65F15, 34H05.

Key words and phrases. structured singular value; spectral value set; block diagonal uncertainties; spectral radius; low-rank matrix manifolds, gradient system of ODEs.

(C)2019 Authors retain the copyrights of their papers, and all open access articles are distributed under the terms of the Creative Commons Attribution License. 
The widely used routine, mussv, available in MATLAB control Toolbox approximates upper bound by means of the diagonal balancing and linear matrix inequalities (LMI) techniques [11, 12]. While on the other hand, lower bound is approximated by generalization of power method [13-14].

In this paper, we present numerical approximations to a lower bound of the SSV associated with mixed real and complex uncertainties. Our approach is based on a two level algorithm, inner-outer algorithm, given in $[17]$.

\section{Overview of the ARticle}

Section 3 provides the basic framework of proposed problem. We explain how a better approximation of the structured singular values can be obtained by a two level algorithm that is an inner-outer algorithm. The outer algorithm determines the perturbation level while the inner algorithm computes the desired perturbation. The inner algorithm then determines a (local) extremizer of the structured spectral value set. In Section 4, we introduce the inner algorithm for the case of pure complex uncertainties. An important characterization of extremizers help us to restrict the problem to a manifold of structured perturbations with normalized and low-rank blocks. A gradient system of ODEs for finding extremizers on this manifold is established. Finally, in Section 5, we give a range of numerical experiments to compare lower bounds of SSV obtained with proposed algorithm [17] to those obtained with mussv.

\section{Framework}

Let $C=\mathbb{C}$ and $R=\mathbb{R}$ and let $C^{n, n}\left(R^{n, n}\right)$ denote the family of complex (real) matrices. For $M \in$ $C^{n, n}\left(R^{n, n}\right)$ we consider an underlying perturbation set with prescribed block diagonal matrices given below

$$
\mathbb{B}=\left\{\operatorname{diag}\left(\delta_{i} I_{i}, \Delta_{j}\right): \delta_{i} \in \mathbb{C}(\mathbb{R}), \Delta_{j} \in \mathbb{C}^{n, n}\left(\mathbb{R}^{n, n}\right)\right\}
$$

The following definition is given in [1], where $\mathrm{I}$ is the $n \times n$ identity matrix.

Definition 3.1. Let $M \in C^{n, n}$ and $\Delta \in \mathbb{B}$ is an admissible perturbation. Then structured singular value of $M$ is denoted by $\mu_{\mathbb{B}}(M)$ and is defined as follows

$$
\mu_{\mathbb{B}}(M):=\frac{1}{\min \left\{\|\Delta\|_{2}: \Delta \in \mathbb{B}, \operatorname{det}(I-M \Delta)=0\right\}} .
$$

In above definition $3.2, \operatorname{det}(\cdot)$ represents the determinant of a matrix $(I-M \Delta)$ while minimum is over an admissible perturbation $\Delta$.

For a general set $\mathbb{B}$ the SSV become smaller and thus we have an upper bound. The important special case, when the set $\mathbb{B}$ only consists of pure complex perturbations, we denote the set by $\mathbb{B}^{*}$ instead of $\mathbb{B}$. For $\Delta \in \mathbb{B}^{*}$ we have $e^{i \theta} \Delta \in \mathbb{B}$ for any $\theta \in \mathbb{R}$. As a result, this gives us a suitable choice of $\Delta \in \Delta_{\mathbb{B}^{*}}$ such that spectral radius attains the maximum value 1 that is $\rho(M \Delta)=1$ iff there is $\Delta^{\prime} \in \Delta_{\mathbb{B}^{*}}$, which possesses the 
same matrix 2-norm so that $M \Delta^{\prime}$ possess an eigenvalue 1 . This in turn implies that the matrix $I-M \Delta^{\prime}$ is singular. This gives us the following alternative definition of the SSV

$$
\mu_{\mathbb{B}^{*}}(M):==\frac{1}{\min \left\{\|\Delta\|_{2}: \Delta \in \mathbb{B}^{*}, \rho(M \Delta)=1\right\}} .
$$

In Equ. (3.3), $\rho(\cdot)$ denotes the spectral radius of a matrix $M \Delta$.

3.1. Overview of the proposed methodology. We need to solve the maximization problem

$$
\lambda(\epsilon)=\arg \max |\lambda|
$$

where maximum is over $\lambda$ for some fixed parameter $\epsilon>0$. It is clear that $\mu_{\mathbb{B}^{*}}(M)$ is the reciprocal of the smallest value of $\epsilon$ for which $\lambda(\epsilon)=1$. This suggests a two level algorithm. In the inner algorithm, we intend to solve the problem addressed in Equ. (3.4). In the outer algorithm we first vary $\epsilon$ by using the fast Newtons method and this exploits the knowledge of the computation of the exact derivative of the extremizers. We address Equ. (3.4) by solving a system of ordinary differential equations.

\section{Computation of LOCAL EXtremizers}

In this section we present the inner algorithm for optimization problem addressed in Equ. (3.4). We use the following standard eigenvalue perturbation result.

Lemma 4.1. [15]. Consider a family of matrices $A: R \rightarrow C^{n, n}$ and let $\lambda(t)$ be an eigenvalue of $A(t)$ for all values of $t$. The eigenvalue $\lambda(t)$ tends to converge to the simple eigenvalue $\lambda_{0}=\lambda(0)$, of $A_{0}=A(0)$ as $t \rightarrow 0$. Therefor $\lambda(t)$ is analytic near $t=0$ with

$$
\frac{d \lambda(t)}{d t}=\frac{X_{0}^{*} B X_{0}}{X_{0}^{*} X_{0}}
$$

where, $X_{0}^{*}, X_{0}$ are the left and right eigenvectors of $A_{0}=A(0)$ associated with simple eigenvalue $\lambda_{0}=\lambda(0)$, that is, $X_{0}^{*}\left(A_{0}-\lambda_{0} I\right)=0$ and $\left(A_{0}-\lambda_{0} I\right) X^{*}=0$ and the matrix $B$ is the time derivative of the matrix valued function $A(t)$.

Definition 4.2. An admissible perturbation $\Delta \in \mathbb{B}^{*}$ such that $\|\Delta\|_{2} \leq 1$ and the matrix $(\epsilon M \Delta)$ for some fixed parameter $\epsilon>0$ has greatest eigenvalue $\lambda_{\max }$ which maximizes locally the modulus of $\Lambda_{\epsilon}^{\mathbb{B}^{*}}(M)$ is known as a local maximizer.

Theorem 4.3. Let

$$
\widehat{\Delta}=\left\{\operatorname{diag}\left(\delta_{i} I_{i}, \Delta_{j}\right): \delta_{i} \in C, \Delta_{j} \in C^{m_{j}, m_{j}}, \forall i=1: S, J=1: F\right\}
$$

with $\|\widehat{\Delta}\|_{2}=1$ be a local extremizer. The matrix $\epsilon M \Delta$ possesses the simple eigenvalue $\lambda=|\lambda| e^{i \theta}, \theta \in R$, with $x$ and $y$ being right and left eigenvectors for an eigenvalue $\lambda$. The eigenvectors are scaled such that $s=e^{i \theta} y^{*} x>0$. Partitioning $x$ and $y$ according to size and structure of $\widehat{\Delta}$ yields

$$
x=\left(x_{1}^{\mathrm{T}}, \ldots, x_{n}^{\mathrm{T}}, x_{n+1}^{\mathrm{T}}, \ldots, x_{n+F}^{\mathrm{T}}\right)^{\mathrm{T}}
$$




$$
y=\left(y_{1}^{\mathrm{T}}, \ldots, y_{n}^{\mathrm{T}}, y_{n+1}^{\mathrm{T}}, \ldots, y_{n+F}^{\mathrm{T}}\right)^{\mathrm{T}}
$$

Now we take $z=M^{*} y=\left(z_{1}^{\mathrm{T}}, \ldots, z_{n}^{\mathrm{T}}, z_{n+1}^{\mathrm{T}}, \ldots, z_{n+F}^{\mathrm{T}}\right)^{\mathrm{T}}$ and assume the non-degeneracy conditions given by

$$
\begin{aligned}
& z_{k}^{*} x_{k} \neq 0 \quad \forall k=1, \ldots, n \\
& \left\|z_{n+h}\right\|_{2} \cdot\left\|x_{n+h}\right\|_{2} \neq 0 \quad \forall h=1, \ldots, F .
\end{aligned}
$$

Hence,

$$
\left|\delta_{k}\right|=1 \quad \forall k=1, \ldots, s \quad \text { and } \quad\|\Delta\|_{2}=1 \quad \forall h=1, \ldots, F
$$

Theorem 4.4. Let

$$
\widehat{\Delta}=\left\{\operatorname{diag}\left(\delta_{i} I_{i}, \Delta_{j}\right): \delta_{i} \in C, \Delta_{j} \in C^{m_{j}, m_{j}}, \forall i=1: S, J=1: F\right\}
$$

with $\|\widehat{\Delta}\|_{2}=1$ be a local extremizer. Suppose that $\lambda, x, z$ are defined and partitioned as in Theorem 4.3 . Assume the non-degeneracy condition of (4.4) and every block possess a singular value which attains the maximum value 1 . Then the matrix,

$$
\widehat{\Delta}=\left\{\operatorname{diag}\left(\delta_{1} I_{1}, \ldots, \delta_{s} I_{s} ; u_{1} v_{1}^{*}, \ldots, u_{F} v_{F}^{*}\right)\right\}
$$

is a local extremizer.

Remark 4.5. Theorem 4.4 allows us to restrict the admissible perturbations in the structured spectral value set given in (3.4) to those with rank-1 blocks. Since the Frobenius and the matrix 2-norms of a rank-1 matrix are same, this helps us to search for extremizers within the sub-manifold

$$
B_{1}^{*}=\left\{\operatorname{diag}\left(\delta_{1} I_{1} ; \Delta_{j}\right): \delta_{i} \in C, \Delta_{j} \in C^{m_{j}, m_{j}}, \forall i=1: S, j=1: F\right\}
$$

4.6. Approximating extremal points of $\Lambda_{\epsilon}^{B^{*}}(M)$.

In order to approximate the local maxima for $\Lambda_{\epsilon}^{B^{*}}(M)$ we construct a matrix valued function $\Delta(t)$ where $\Delta(t) \in \mathbb{B}^{*}$ such that the largest eigenvalue $\lambda$ of the matrix $\epsilon M \Delta(t)$ achieves the maximum (local) growth. We then derive a gradient system of ODEs which satisfies the choice of the initial matrix admissible perturbation $\Delta(t)$.

4.7. The local optimization problem.

We consider the fact that $\lambda=|\lambda| e^{i \theta}$ is the simple eigenvalue with the corresponding eigenvectors, normalized such that

$$
\|x\|=\|y\|=1, \quad y^{*} x=\left|y^{*} x\right| e^{-i \theta}
$$

As a result of the Lemma 4.1, we get the following expression for the change in the largest eigenvalue.

$$
\frac{d}{d t}|\lambda|^{2}=\frac{2|\lambda|}{\left|y^{*} x\right|} \operatorname{Re}\left(z^{*} \dot{\Delta} x\right), z=M^{*} y
$$


The eigenvectors $x$ and $y$ are defined and normalized as in the Theorem 4.3. Now, by considering the suitable perturbation $\Delta \in B_{1}^{*}$ with $B_{1}^{*}$ in Equ. (4.6), we aim to determine a direction $\Delta^{*}=z$ that (locally) maximizes the increase of the modulus of $\lambda$. This amounts to determining a direction $Z$ as given in the following equation

$$
Z=\operatorname{diag}\left(\omega_{1} I_{r_{1}}, \ldots, \omega_{s} I_{r_{N}}, \Omega_{1}, \ldots, \Omega_{F}\right)
$$

which is the solution of the following optimization problem

$$
\begin{gathered}
Z=\arg \max \left\{\operatorname{Re}\left(z^{*} Z x\right)\right\} \\
\text { subject to } \operatorname{Re}\left(\bar{\delta}_{i} \omega_{i}\right)=0, i=1: S, \\
\text { and } \operatorname{Re}\left\langle\Delta_{j}, \Omega_{j}\right\rangle=0, j=1: F .
\end{gathered}
$$

The linear constraints in maximization problem in Equ. (4.10) ensure that $Z$ lies in the tangent space of the manifold $B_{1}^{*}$ at $\Delta(t)$. In particular Equ. (4.10) ensures that the norm of each block of the admissible perturbation $\Delta(t)$ remain conserved.

Lemma 4.6. The solution of the maximization problem as discussed in (4.10) is given by

$$
Z_{*}=\left\{\operatorname{diag}\left(\omega_{1} I_{r_{1}}, \ldots, \omega_{N} I_{r_{N}}, \Omega_{1}, \ldots, \Omega_{F}\right)\right\}
$$

with

$$
\begin{aligned}
\omega_{i} & =\nu_{i}\left(x_{i}^{*} z_{i}-\operatorname{Re}\left(x_{i}^{*} z_{i} \bar{\delta}_{i}\right) \delta_{i}\right), \quad i=1, \ldots, S \\
\Omega_{j} & =\zeta_{j}\left(z_{s+j} x_{N+j}^{*}-\operatorname{Re}\left\langle\Delta_{j}, z_{s+j} x_{s+j}^{*}\right\rangle \Delta_{j}\right), \quad j=1, \ldots, F .
\end{aligned}
$$

Where $v_{i}>0$ and $\zeta_{j}>0$. If the right-hand sides are different from zero then $Z \in B_{1}^{*}$.

Corollary 4.7. The result of the lemma 4.6 can be written as follows:

$$
Z_{*}=D_{1} P_{\mathbb{B}^{*}}\left(z x^{*}\right)-D_{2} \Delta
$$

where $P_{\mathbb{B}^{*}}(\cdot)$ is the orthogonal projection and $D_{1}, D_{2} \in \mathbb{B}^{*}$ are diagonal matrices with $D_{1}$ is positive.

4.8. The gradient system of ordinary differential equations.

Following Lemma 4.6 and Corollary 4.7 we consider the following differential equations on the manifold $B_{1}^{*}$.

$$
\dot{\Delta}=D_{1} P_{\mathbb{B}^{*}}\left(z x^{*}\right)-D_{2} \Delta .
$$

where, $x(t)$ is an eigenvector associated to a simple eigenvalue $\lambda(t)$ of the matrix $\epsilon M \Delta(t)$ for some fixed $\epsilon>0$. Also note that the quantities $z(t), D_{1}$ and $D_{2}$ depend on the choice of the matrix valued function that is $\Delta(t)$ as well. The differential Equ. (4.15) is a gradient system of ODEs. 
4.9. Choice of initial value matrix and $\epsilon_{0}$.

In order to compute $\epsilon_{0}$ we choose the initial value matrix

$$
\Delta_{0}=D P_{\Delta_{\mathbb{B}}}\left(w v^{*}\right)
$$

where $D$ is the positive diagonal matrix such that $\Delta_{0} \in \mathbb{B}^{*}$. As a natural choice for the initialization of the perturbation level, we take $\epsilon_{0}$ as

$$
\epsilon_{0}=\frac{1}{\widehat{\mu}_{\mathbb{B}^{*}}(M)}
$$

where $\widehat{\mu}_{\mathbb{B}^{*}}(M)$ is the upper bound of $\mu$-value approximated by mussv.

\section{Numerical Testing}

In this section we present various numerical experimentations for both pure and mixed real and complex perturbations. The comparisons of lower bounds of structured singular values for a class of matrices obtained for DC Motor are considered.

Example 1. Consider the following five dimensional matrix.

$$
M=\left[\begin{array}{ccccc}
-.050-.113 i & -.012-.028 i & -.216+.072 i & -.000-.000 i & 6.306-2.099 i \\
.113-.050 i & .028-.012 i & -.072-.216 i & .000-.000 i & 2.099+6.306 i \\
-.050-.113 i & -.012-.028 i & -.073+.072 i & -.000-.000 i & 6.306-2.099 i \\
-.004-.008 i & -.001-.002 i & .001+.005 i & .142-.000 i & -.042-.153 i \\
-.004-.008 i & -.001-.002 i & -.001-.002 i & -.000-.000 i & -.042-.153 i
\end{array}\right]
$$

Also, consider the set of block diagonal uncertainties as an input argument. The uncertainty set is taken as

$$
\mathbb{B}=\left\{\operatorname{diag}\left(\delta_{1} I_{2}, \Delta_{1}\right): \delta_{1} \in \mathbb{R}, \Delta_{1} \in \mathbb{C}^{3,3}\right\}
$$

Making use of MATLAB function mussv, we obtain an admissible perturbation set $\widehat{\Delta}$, which is given below

$$
\widehat{\Delta}=\left[\begin{array}{ccccc}
-.149 & 0 & 0 & 0 & 0 \\
0 & -.149 & 0 & 0 & 0 \\
0 & 0 & -.001-.001 i & 0 & 0 \\
0 & 0 & .000-.0001 i & 0 & 0 \\
0 & 0 & -.143-.044 i & -.0008+0.0033 i & -.0008+.0033 i
\end{array}\right]
$$

The 2-norm of admissible perturbation is obtained as 0.1499 while the lower bound of structured singular value is obtained as $\mu_{P D}^{l}(M)=6.6709$ and an upper bound $\mu_{P D}^{u}(M)=6.6715$. 
Applying the algorithm presented in article [17], we obtain the admissible uncertainty $\epsilon \widehat{\nabla}$ with $\epsilon=0.1499$ and $\widehat{\nabla}$ with

$$
\widehat{\nabla}=\left[\begin{array}{ccccc}
-1 & 0 & 0 & 0 & 0 \\
0 & -1 & 0 & 0 & 0 \\
0 & 0 & -0.0115-0.0100 i & 0.0002-0.0002 i & 0.0002-0.0002 i \\
0 & 0 & 0.0001-0.0003 i & 0 & 0 \\
0 & 0 & -0.9541-0.2976 i & -0.0065+0.0207 i & -0.0070+0.0206 i
\end{array}\right]
$$

In this case the admissible uncertainty has a unit 2-norm while the obtained lower bound of structured singular value is $\mu_{\text {Our }}^{l}(M)=6.6708$.

Example 2. Consider the following five dimensional matrix.

$$
M=\left[\begin{array}{ccccc}
-.169-.155 i & -.042-.038 i & -.176+.137 i & -.000-.000 i & 5.136-3.998 i \\
.310-.338 i & .077-.084 i & -.274-.352 i & .001-.001 i & 7.997+10.272 i \\
-.169-.155 i & -.042-.038 i & -.033+.137 i & -.000-.000 i & 5.136-3.998 i \\
-.014-.008 i & -.003-.002 i & .005+.009 i & .142-.000 i & -.168-.266 i \\
-.014-.008 i & -.003-.002 i & .005-.009 i & .142-.000 i & -.168-.266 i
\end{array}\right]
$$

Also, consider the set of block diagonal uncertainties as an input argument. The uncertainty set is taken as

$$
\mathbb{B}=\left\{\operatorname{diag}\left(\delta_{1} I_{1}, \Delta_{1}\right): \delta_{1} \in \mathbb{R}, \Delta_{1} \in \mathbb{C}^{4,4}\right\}
$$

Making use of MATLAB function mussv, we obtain an admissible perturbation set $\widehat{\Delta}$, which is given below

$$
\widehat{\Delta}=\left[\begin{array}{ccccc}
-0.0679 & 0 & 0 & 0 & 0 \\
0 & -.0004+.0004 i & -.0002+.0002 i & 0 & 0 \\
0 & -.0013+.0014 i & -.0007-.0006 i & 0 & 0 \\
0 & 0 & 0 & 0 & 0 \\
0 & .036-.048 i & -.024+.018 i & -.0007+.001 i & -.0007+.001 i
\end{array}\right]
$$

The 2-norm of admissible perturbation is obtained as 0.1499 while the lower bound of structured singular value is obtained as $\mu_{P D}^{l}(M)=14.7375$ and an upper bound $\mu_{P D}^{u}(M)=14.7988$. 
Applying the algorithm presented in article [17], we obtain the admissible uncertainty $\epsilon \widehat{\nabla}$ with $\epsilon=0.0679$ and $\widehat{\nabla}$ with

$$
\widehat{\nabla}=\left[\begin{array}{ccccc}
-1 & 0 & 0 & 0 & 0 \\
0 & .005+.005 i & -.002+.002 i & -.0001-.0001 i & -.0001-.0001 i \\
0 & -.018+.020 i & -.010-.009 i & .0004-.0005 i & .0004-.0005 i \\
0 & -.0001+.0001 i & 0 & 0 & 0 \\
0 & .541+.711 i & .355+.270 i & -.0109+.018 i & -.010+.181 i
\end{array}\right]
$$

In this case the admissible uncertainty has a unit 2-norm while the obtained lower bound of structured singular value is $\mu_{\text {Our }}^{l}(M)=14.7375$.

Example 3. Consider the following five dimensional matrix.

$$
M=\left[\begin{array}{ccccc}
-.283-.099 i & -.070-.024 i & -.109+.178 i & -.001-.000 i & 3.195-5.200 i \\
.299-.849 i & .074-.212 i & -.534-.328 i & .001-.003 i & 15.600+9.587 i \\
-.283-.099 i & -.070-.024 i & -.033+.178 i & -.001-.000 i & 3.195-5.200 i \\
-.021-.001 i & -.005-.0003 i & .011+.009 i & .142-.000 i & -.346-.286 i \\
-.021-.001 i & -.005-.0003 i & .011+.009 i & -.0001-.000 & -.346-.286 i
\end{array}\right]
$$

Also, consider the set of block diagonal uncertainties as an input argument. The uncertainty set is taken as

$$
\mathbb{B}=\left\{\operatorname{diag}\left(\delta_{1} I_{1}, \delta_{2} I_{1}, \delta_{3} I_{1}, \Delta_{1}\right): \delta_{1}, \delta_{2}, \delta_{3} \in \mathbb{R}, \Delta_{1} \in \mathbb{C}^{2,2}\right\}
$$

Making use of MATLAB function mussv, we obtain an admissible perturbation set $\widehat{\Delta}$, which is given below

$$
\widehat{\Delta}=\left[\begin{array}{ccccc}
-.586+1.067 i & 0 & 0 & 0 & 0 \\
0 & -.586+1.067 i & 0 & 0 & 0 \\
0 & 0 & -.586+1.067 i & 0 & 0 \\
0 & 0 & 0 & .109 & .105 \\
0 & 0 & 0 & -.594+.631 i & -.576+.612 i
\end{array}\right]
$$

The 2-norm of admissible perturbation is obtained as 1.2176 while the lower bound of structured singular value is obtained as $\mu_{P D}^{l}(M)=0.8213$ and an upper bound $\mu_{P D}^{u}(M)=0.8213$. 
Applying the algorithm presented in article [17], we obtain the admissible uncertainty $\epsilon \widehat{\nabla}$ with $\epsilon=1.2176$ and $\widehat{\nabla}$ with

$$
\widehat{\nabla}=\left[\begin{array}{ccccc}
-.492+.870 & 0 & 0 & 0 & 0 \\
0 & -.492+.870 & 0 & 0 & 0 \\
0 & 0 & -.492+.870 & 0 & 0 \\
0 & 0 & 0 & .087+.0009 i & .085+.001 i \\
0 & 0 & 0 & -.484+.520 i & -.472+.506 i
\end{array}\right]
$$

In this case the admissible uncertainty has a unit 2-norm while the obtained lower bound of structured singular value is $\mu_{\text {Our }}^{l}(M)=0.8213$.

Example 4. Consider the following five dimensional matrix.

$$
M=\left[\begin{array}{ccccc}
-.326+.009 i & -.081-.002 i & -.036+.181 i & -.001-.000 i & 1.065-5.282 i \\
-.038-1.306 i & -.009-.326 i & -.724-.146 i & -.0002-.005 i & 21.128+4.260 i \\
-.326+.009 i & -.081+.002 i & .106+.181 i & -.001 & 1.065-5.282 i \\
-.020+.009 i & -.005+0.002 i & .017+.006 i & .142-.000 i & -.498-.196 i \\
-.020+.009 i & -.005+.002 i & .017+.006 i & -.0001-.000 i & -.498-0.196 i
\end{array}\right]
$$

Also, consider the set of block diagonal uncertainties as an input argument. The uncertainty set is taken as

$$
\mathbb{B}=\left\{\operatorname{diag}\left(\delta_{1} I_{1}, \delta_{2} I_{1}, \Delta_{1}\right): \delta_{1}, \delta_{2}, \in \mathbb{R}, \Delta_{1} \in \mathbb{C}^{3,3}\right\}
$$

Making use of MATLAB function mussv, we obtain an admissible perturbation set $\widehat{\Delta}$, which is given below

$$
\widehat{\Delta}=\left[\begin{array}{ccccc}
-.173 & 0 & 0 & 0 & 0 \\
0 & -.134 & 0 & 0 & 0 \\
0 & 0 & .002-.005 i & .0006+.0002 i & .0006+.0002 i \\
0 & 0 & -.0004 i & 0 & 0 \\
0 & 0 & .041+.166 i & -.014+.006 i & -.014+.006 i
\end{array}\right]
$$

The 2-norm of admissible perturbation is obtained as 0.1734 while the lower bound of structured singular value is obtained as $\mu_{P D}^{l}(M)=5.7675$ and an upper bound $\mu_{P D}^{u}(M)=5.7786$. 
Applying the algorithm presented in article [17], we obtain the admissible uncertainty $\epsilon \widehat{\nabla}$ with $\epsilon=2.6959$ and $\widehat{\nabla}$ with

$$
\widehat{\nabla}=\left[\begin{array}{ccccc}
-1 & 0 & 0 & 0 & 0 \\
0 & -.911 & 0 & 0 & 0 \\
0 & 0 & .015-.032 i & .003+.0009 i & .003+.0009 i \\
0 & 0 & .0002-.002 i & .0002 & .0002 \\
0 & 0 & .247+.959 i & -.083+.038 i & -.083+.038 i
\end{array}\right]
$$

In this case the admissible uncertainty has a unit 2-norm while the obtained lower bound of structured singular value is $\mu_{\text {Our }}^{l}(M)=0.3709$.

In each of following figure, we present the comparison of lower bounds of structured singular values obtained by MATLAB routine mussv and the algorithm presented in [17], for five dimensional complex matrices. Each of these matrix is computed from [16].

\section{Conclusion}

In this article we have considered the problem for the computation of the lower bound of structured singular values for a family of complex matrices obtained in [16]. The computation of structured singular values plays an important role in robust stability and instability in control. The experimental results show the comparison of the lower bounds computed by algorithm [17] when compared to well-known1 MATLAB function mussv available in MATLAB Control toolbox.

\section{Nomenclature}

$\mathbb{B} \quad$ Family of block diagonal matrices

$\epsilon_{0} \quad$ Perturbation level

$\Delta_{0} \quad$ Initial admissible perturbation

$\mu \quad$ Structured Singular Values

\section{REFERENCES}

[1] Packard, Andrew and Doyle, John. The complex structured singular value. Automatica, 29(1993): 71-109.

[2] Bernhardsson, Bo and Rantzer, Anders and Qiu, Li. Real perturbation values and real quadratic forms in a complex vector space. Linear Algebra Appl., 270(1998): 131-154.

[3] Chen, Jie and Fan, Michael KH and Nett, Carl N. Structured singular values with nondiagonal structures. I. Characterizations. IEEE Trans. Automatic Control, 41(1996): 1507-1511.

[4] Chen, Jie and Fan, Michael KH and Nett, Carl N. Structured singular values with nondiagonal structures. II. Computation. IEEE Trans. Automatic Control, 41(1996): 1511-1516. 
[5] Hinrichsen, Diederich and Pritchard, Anthony J. Mathematical systems theory I: modelling, state space analysis, stability and robustness. Vol. 48. Berlin: Springer, 2005.

[6] Karow, Michael. $\mu$-values and spectral value sets for linear perturbation classes defined by a scalar product. SIAM J. Matrix Anal. Appl., 32(2011): 845-865.

[7] Karow, Michael and Hinrichsen, Diederich and Pritchard, Anthony J. Interconnected systems with uncertain couplings: Explicit formulae for mu-values, spectral value sets, and stability radii. SIAM J. Control Optim., 45(2006): 856-884.

[8] Qiu, Li and Bernhardsson, Bo and Rantzer, Anders and Davison, Edward J and Doyle, JC. A formula for computation of the real stability radius, Automatica, 31(1995): 879890 .

[9] Zhou, Kemin and Doyle, John Comstock and Glover, Keith and others. Robust and optimal control. Prentice hall New Jersey, Volume 40: (1996).

[10] Braatz, Richard P and Young, Peter M and Doyle, John C and Morari, Manfred. Computational complexity of $\mu$ calculation. IEEE Trans. Automatic Control, 39(1994): 1000-1002.

[11] Young, Peter M and Newlin, Matthew P and Doyle, John C. Practical computation of the mixed $\mu$ problem. American Control Conference : 2190-2194 (1992)

[12] Fan, Michael KH and Tits, Andre L and Doyle, John C. Robustness in the presence of mixed parametric uncertainty and unmodeled dynamics. IEEE Trans. Automatic Control, 36(1991): 25-38.

[13] Young, Peter M and DOYLE, John C and PACKARD, Andy and others. Theoretical and Computational Aspects of the Structured Singular Value. Syst. Control Inf., 38(1994): 129-138.

[14] Packard, Andy and Fan, Michael KH and Doyle, John. A power method for the structured singular value. Decision and Control, 1988., Proceedings of the 27th IEEE Conference on, 2132-2137 (1988).

[15] Kato, T. Perturbation Theory for Linear Operators, Classics in Mathematics (Springer-Verlag, Berlin, 1995). Reprint of the 1980 edition, (1980).

[16] Fabrizi, Andrea and Roos, Clement and Biannic, Jean-Marc. A detailed comparative analysis of $\mu$ lower bound algorithms. European Control Conference 2014, (2014).

[17] Rehman, Mutti-Ur and Tabassum, Shabana. Numerical Computation of Structured Singular Values for Companion Matrices. J. Appl. Math. Phys., 5(2017): 1057-1072. 


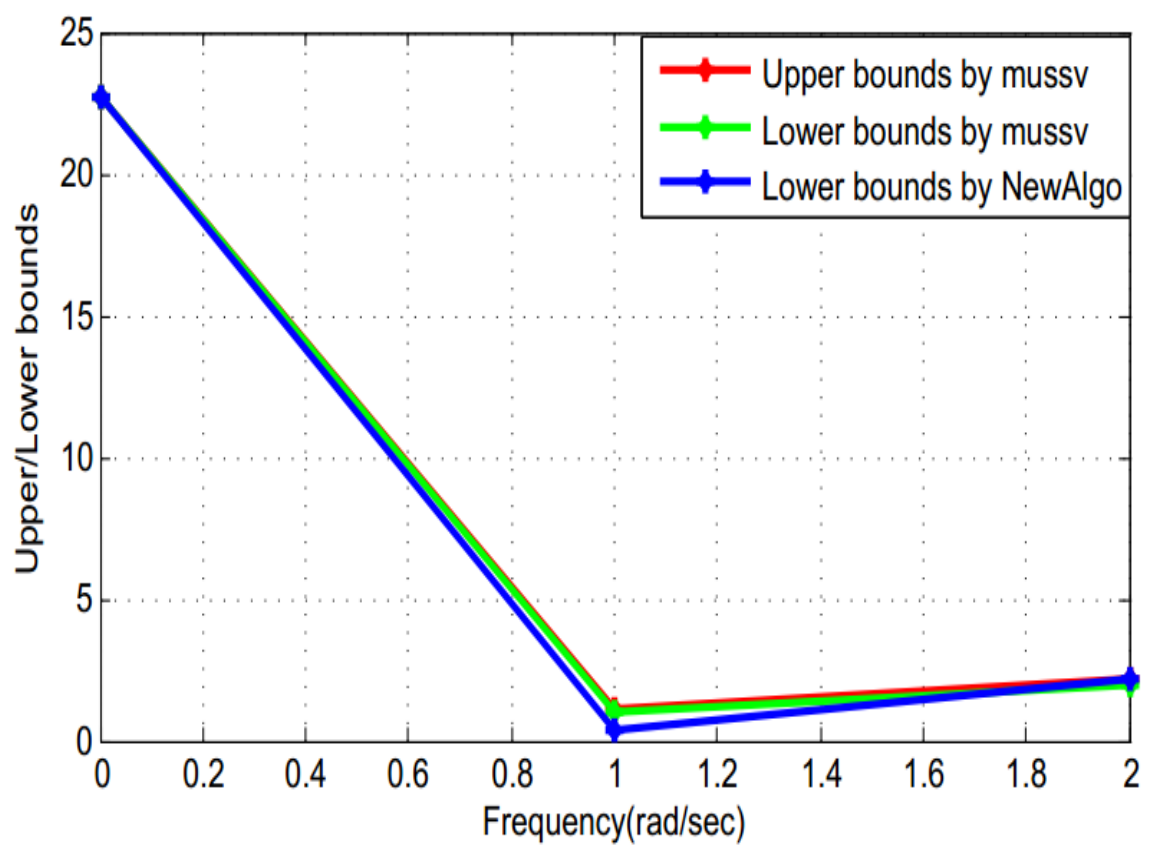

Figure 1. The comparison of lower

bounds of structured singular values for the frequency $=1,2,3$.

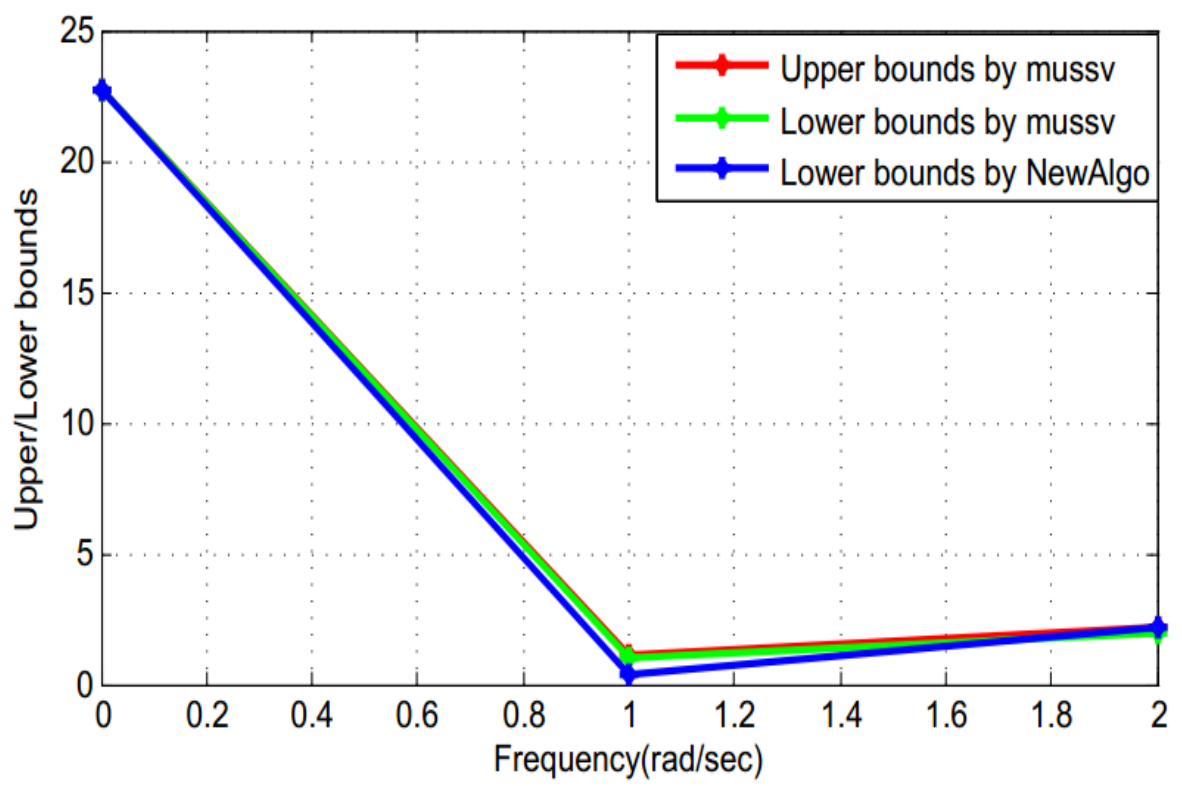

Figure 2. The comparison of lower bounds of structured singular values for the frequency $=1,2,3$. 


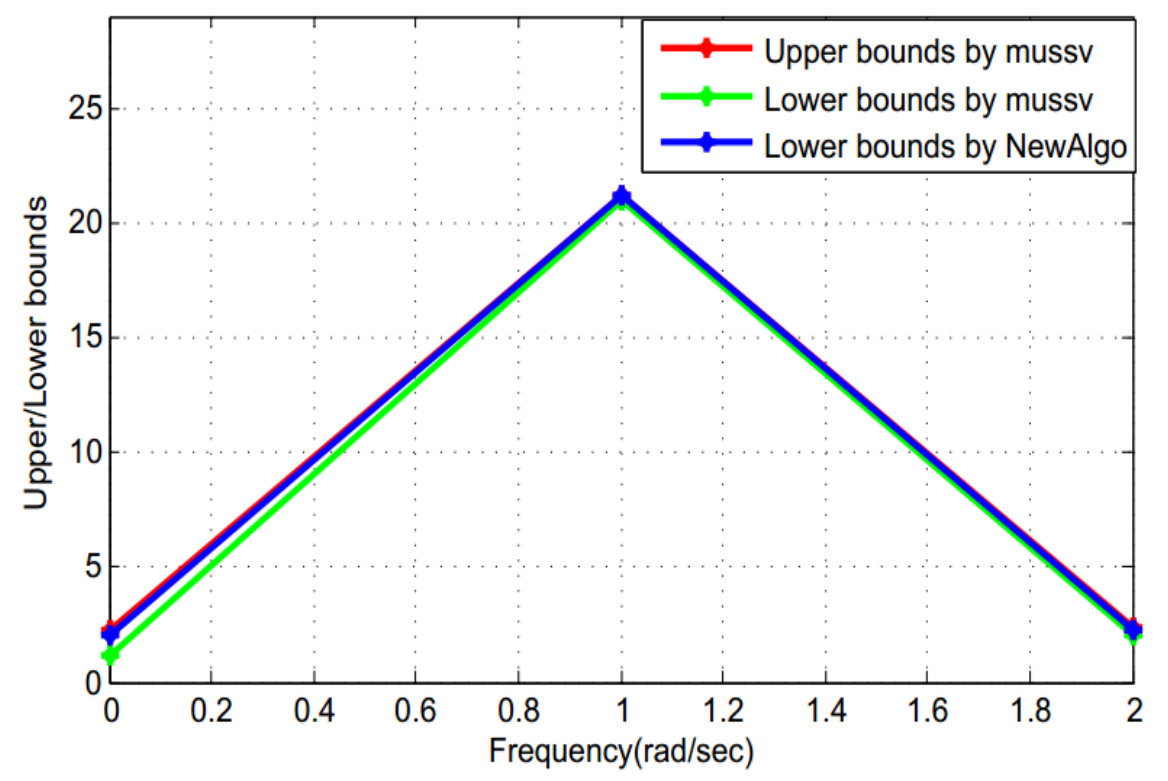

Figure 3. The comparison of lower

bounds of structured singular values

for the frequency $=1,2,3$.

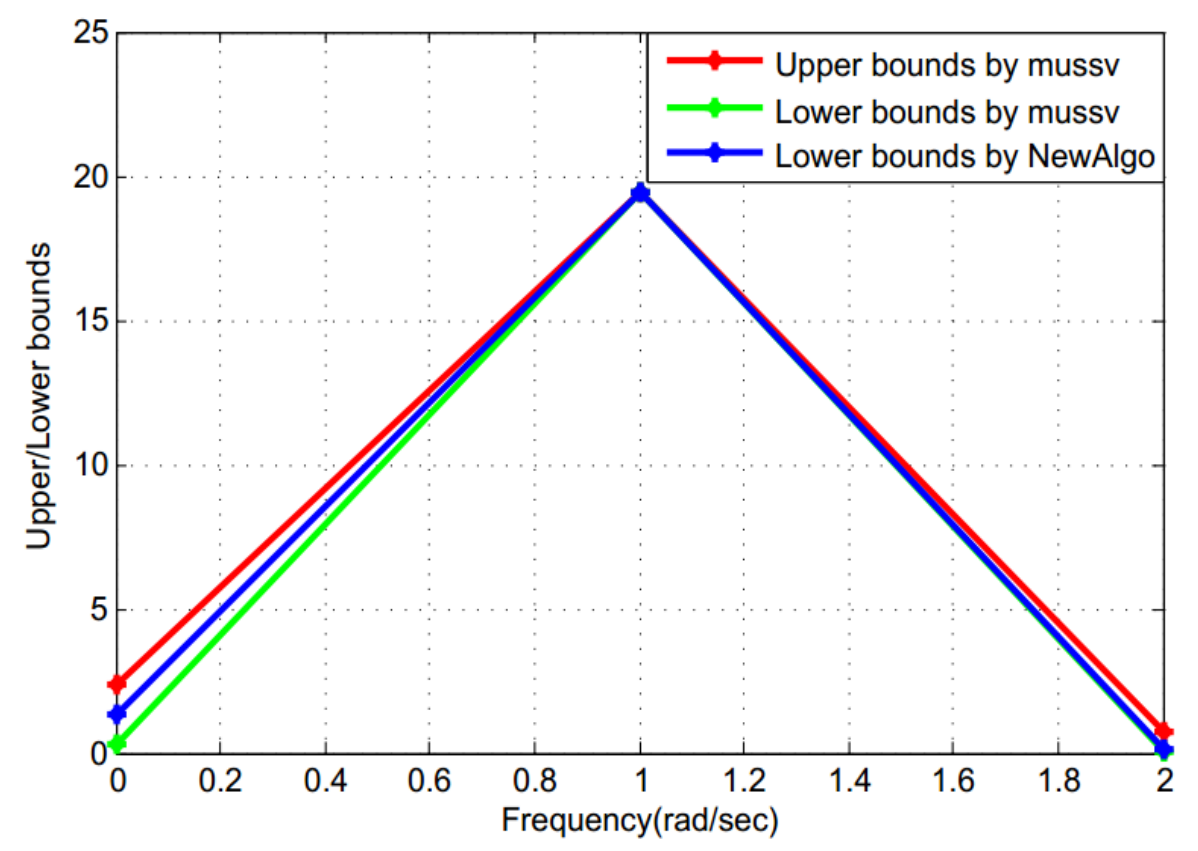

Figure 4. The comparison of lower

bounds of structured singular values

for the frequency $=1,2,3$. 


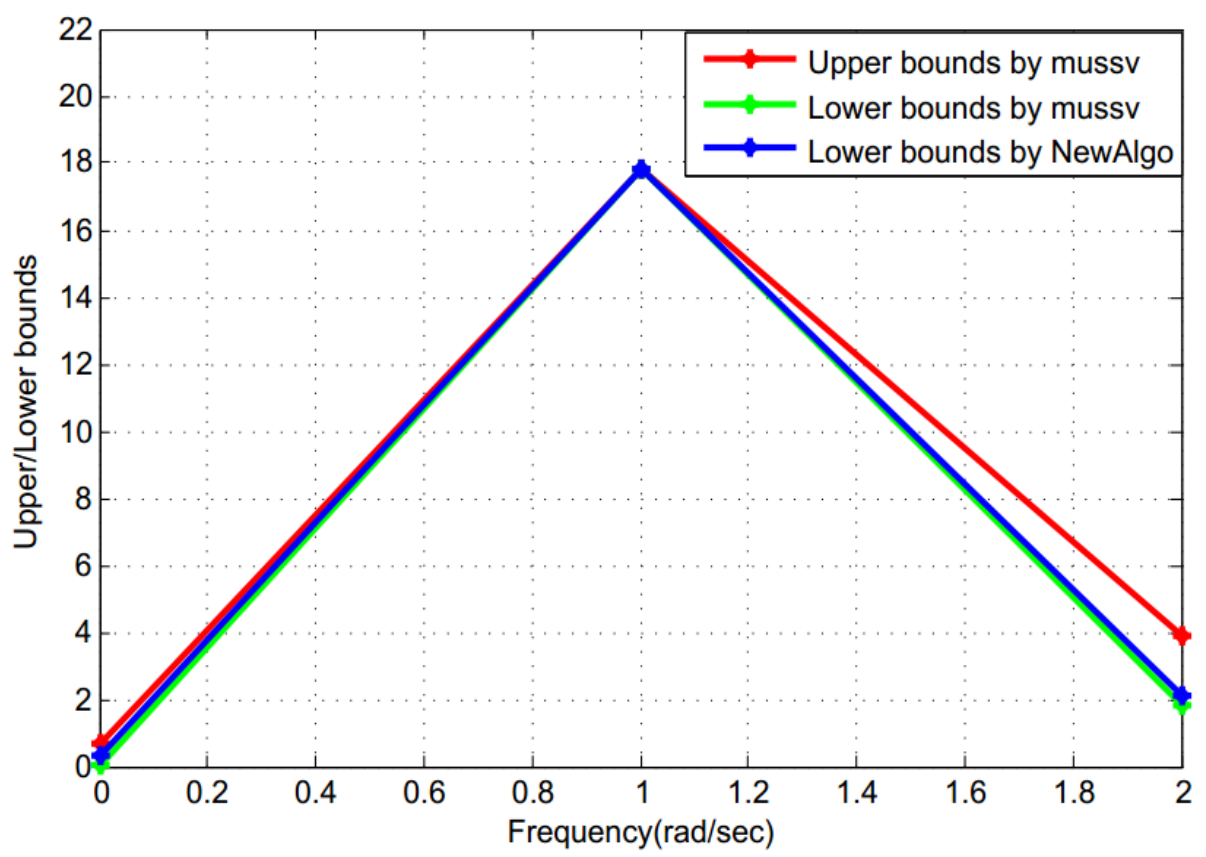

FiguRE 5. The comparison of lower

bounds of structured singular values for the frequency $=1,2,3$.

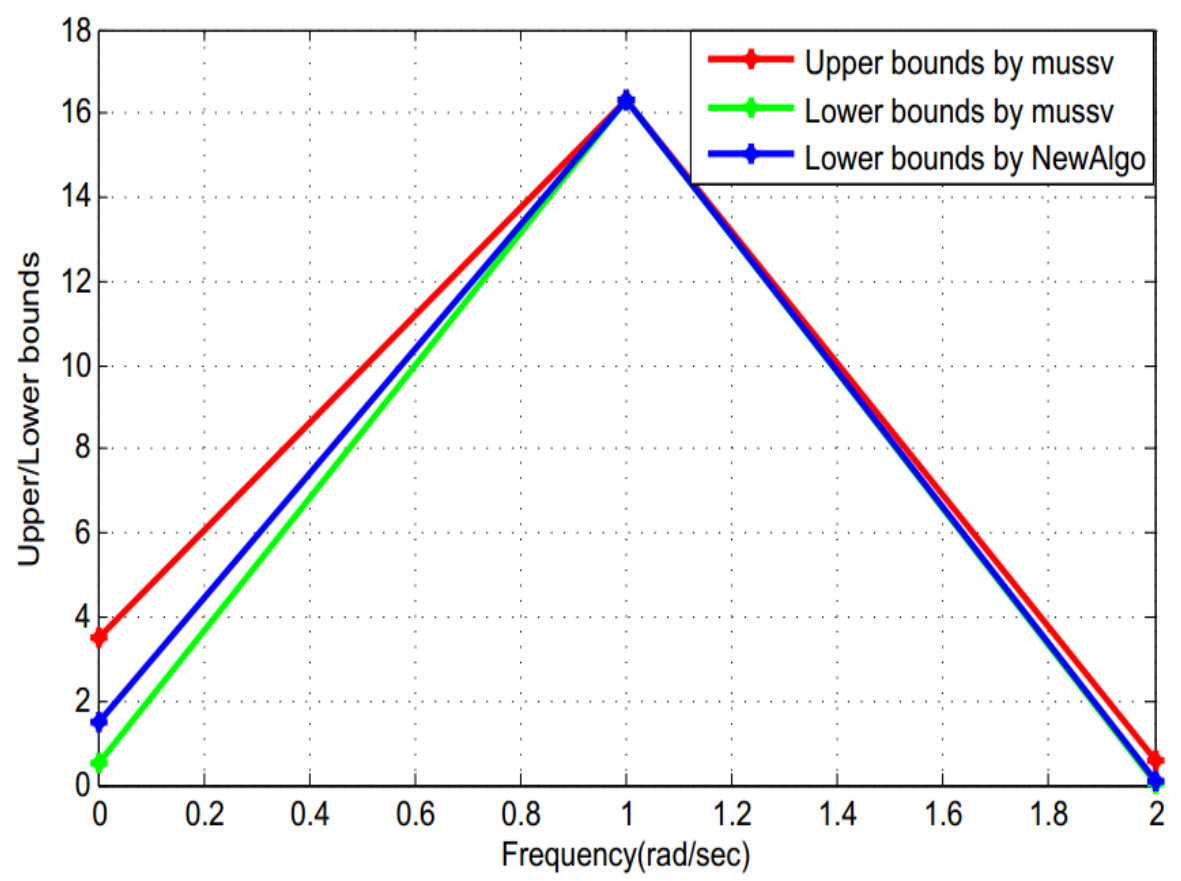

Figure 6. The comparison of lower bounds of structured singular values for the frequency $=1,2,3$. 